\title{
Highly Sensitive Detection of Nitro compounds Using Fluorescent Copolymer Based FRET System
}

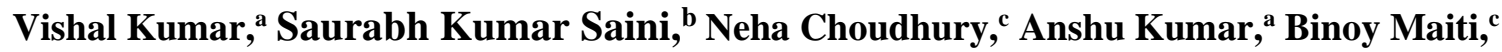 \\ Priyadarsi De, ${ }^{\mathrm{c}}$ Mahesh Kumar, ${ }^{\mathrm{b}}$ and Soumitra Satapathi*a
}

aDepartment of Physics, Indian Institute of Technology Roorkee, Roorkee, Uttarakhand, 247667, India.

${ }^{\mathrm{b}}$ Advanced Materials \& Device Metrology Division, National Physical Laboratory, New Delhi 110012.

'Polymer Research Centre and Centre for Advanced Functional Materials, Department of Chemical Sciences, Indian Institute of Science Education and Research Kolkata, Mohanpur, 741246 Nadia, West Bengal, India.

*Corresponding Author: soumitra.satapathi@ph.iitr.ac.in 


\section{Supporting Information}

\section{Table of Content}

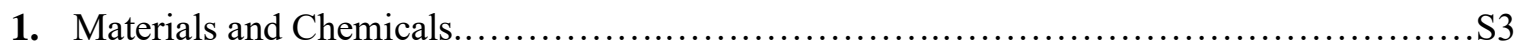

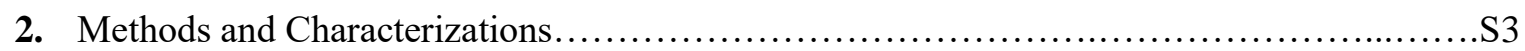

3. Synthesis and structural characterization of tryptophan-based copolymer P(DMA-co-BTE) (RP). S4

4. Synthesis and structural characterization of dansyl-tagged copolymer P(MMA-co-(AlaHEMA)-co-(Dansyl-Ala-HEMA)) (DCP). . .55

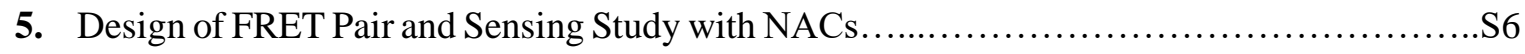

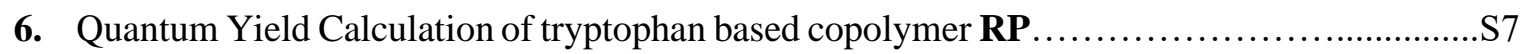

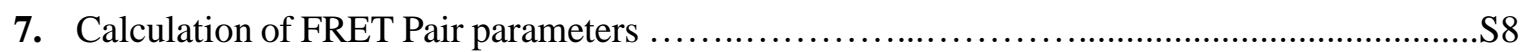

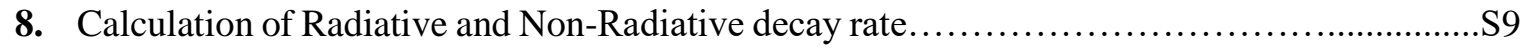

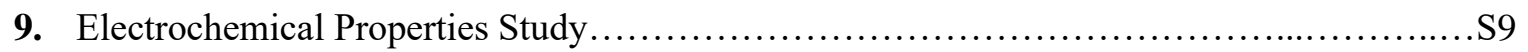

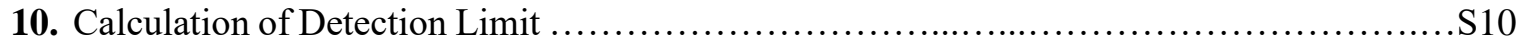

11. Ultrafast Transient Absorption (TA) spectroscopic study .............................. 12

12. Visual Detection of NACs and Its Reversibility........................................ 13

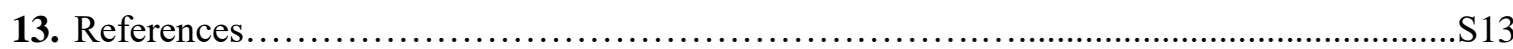




\section{Supporting Information}

\section{Materials and Chemicals}

Boc-L-tryptophan (Boc-L-Trp-OH, 99\%) and trifluoroacetic acid (TFA, 99.5\%) were procured from Sisco Research Laboratories Pvt. Ltd. (SRL), India and utilised as received. Dicyclohexylcabodiimide (DCC, 99\%), 4dimethylaminopyridine (DMAP, 99\%), 2,2'-azobis-(2-methylpropionitrile) (AIBN), anhydrous $N, N^{\prime}-$ dimethylformamide (DMF, 99.9\%), N,N-dimethylacrylamide (DMA, 99\%), 2-hydroxyethyl methacrylate (HEMA, 97\%), Boc-L-alanine (Boc-L-Ala-OH, 99\%) and dansyl chloride (98\%) were procured from SigmaAldrich and utilized without further purification, except for AIBN and DMA. AIBN was used after recrystallization in methanol, and DMA was purified through a basic alumina column prior to use. The 4-cyano4-[(dodecylsulfanylthiocarbonyl)sulfanyl]pentanoic acid (CDP) was synthesized using the literature procedure. ${ }^{1}$ Methyl methacrylate (MMA) (Sigma, 99\%) was passed through a basic alumina column prior to polymerization. The tryptophan based vinyl monomer, Boc-tryptophan methacryloyloxyethyl ester (Boc-Trp-HEMA, BTE), was synthesized by the esterification reaction of Boc-Trp-OH with HEMA in the presence of DCC and DMAP as reported previously. ${ }^{2}$ Similarly, alanine based vinyl monomer, Boc-alanine methacryloyloxyethyl ester (Boc-AlaHEMA) was synthesized by DCC/DMAP esterification reaction of Boc-Ala-OH with HEMA. ${ }^{3}$ The NMR solvent $\mathrm{CDCl}_{3}(99.8 \% \mathrm{D}), \mathrm{D}_{2} \mathrm{O}(99 \% \mathrm{D})$ and dimethylsulfoxide- $d_{6}\left(\mathrm{DMSO}-d_{6}\right)(99.8 \% \mathrm{D})$ were procured from Cambridge Isotope Laboratories, Inc., USA. Carbazole (95\%) was procured from Laboratory Reagents \& Fine Chemicals (LOBA Chemie), India and used as received. The solvents, for example, tetrahydrofuran (THF), dichloromethane (DCM), methanol (MeOH), ethyl acetate (EtOAc), chloroform hexanes (mixture of isomers) and acetone were purified by following standard procedures.

\section{Methods and Characterizations}

The ${ }^{1} \mathrm{H}$ NMR spectra were recorded on a Bruker Avance ${ }^{\mathrm{III}} 500$ spectrometer, operated at $500 \mathrm{MHz}$. Size Exclusion Chromatography (SEC) was used to get an estimated value of dispersity $(\bigoplus)$ and molecular weight of the polymers in DMF solvent with $0.8 \mathrm{~mL} / \mathrm{min}$ flow rate at $40{ }^{\circ} \mathrm{C}$. Our SEC instrument is facilitated with the following components: Waters 1515 HPLC pump, Waters 2414 Refractive Index (RI) detector, one styragel HR3 column $(7.8 \times 300 \mathrm{~mm})$ and one styragel HR 4E column $(7.8 \times 300 \mathrm{~mm})$. Additionally, for calibrating the instrument, Poly(methyl methacrylate) (PMMA) standards were employed. Further, to record all absorbance, excitation and emission spectra, we operate LASANY LI-2800 UV-Vis double beam spectrophotometer and SHIMANDZU RF-6000 Spectro Fluorophotometer were used. 


\section{Supporting Information}

The time correlated single photon counting (TCSPC) measurements were performed on Horiba Jobin Yvon, Florocube with Excitation Sources: Nano LEDs with a wavelength of $340 \mathrm{~nm}$ for DCP and $295 \mathrm{~nm}$ for RP and FRET pair. The fluorescence decay signals were collected at TBX-04D photomultiplier at the emission wavelength of the donor (RP) and analyzed using equation $(\mathrm{S} 1)^{3}$

$$
I(\mathrm{t})=\sum_{i}^{n} \alpha_{i} \exp \left(-t / \tau_{i}\right)
$$

Here, $\mathrm{n}$ is the number of emissive units, and $\alpha_{i}$ and $\tau_{i}$ are the pre-exponential factors and the excited-state fluorescence decay time associated with the $i$ th component, respectively. The typical average decay time, $\langle\tau\rangle$, is determined from equation (S2)

$$
\langle\tau\rangle=\sum_{i=1}^{n} A_{i} \tau_{i}
$$

where $A_{i}\left(=\alpha_{i} / \sum \alpha_{i}\right)$ is the contribution of the decay component.

The ultrafast transient absorption (TA) measurements were performed on Helios pump-probe ultrafast spectroscopy system comprising of Ti:sapphire based laser oscillator (Coherent Micra), Amplifier (Coherent legend), optical parametric amplifier (OPA) (TOPAS) with BBO (Barium Borate) crystal. The amplifier produces a Gaussian-shaped laser pulses of $\sim 35 \mathrm{fs}$ centred at $800 \mathrm{~nm}$ with an average power of $3.5 \mathrm{~W}(1 \mathrm{kHz})$. The amplified laser beam is split into two parts whose $70 \%$ is fed to the OPA, while other part $30 \%$ is fed to the spectrometer via a delay stage capable of 0-8 ns and used as a probe beam. The white-light continuum (WLC) of probe beam (400-850 nm) was generated by passing the splitted beam through sapphire plate. A highly stable OPA output of $\sim 350 \mathrm{~nm}$ was selected as a pump beam with a fluence of $\sim 50 \mu \mathrm{J} / \mathrm{cm}^{2}$. Both the pump and probe beams are focused on the sample, ensuring spatial overlapping. The surface Xplorer software was used for data analysis and fitting, while the spectral resolution was calibrated with the dye ZnTPP and the ocean optics spectrometer calibrated inhouse.

\section{Synthesis of tryptophan-based copolymer, $\operatorname{Poly}[(N, N$-dimethylacrylamide)-co-(Boc- Trp-EMA)], P(DMA-co-BTE), RP}

The desired copolymer, RP was synthesized by copolymerizing DMA and BTE in DMF at $70{ }^{\circ} \mathrm{C}$ by using $\mathrm{CDP}$ as the chain transfer agent and AIBN as the radical source with $[\mathrm{DMA}+\mathrm{BTE}] /[\mathrm{CDP}] /[\mathrm{AIBN}]=150$ : 1: 0.05 following a well-established Reversible addition-fragmentation chain-transfer (RAFT) copolymerization procedure. $^{2}$ The feed ratio of $[\mathrm{DMA}] /[\mathrm{BTE}]=138: 12$ was used in the polymerization reaction. ${ }^{2,4}$ 


\section{Supporting Information}
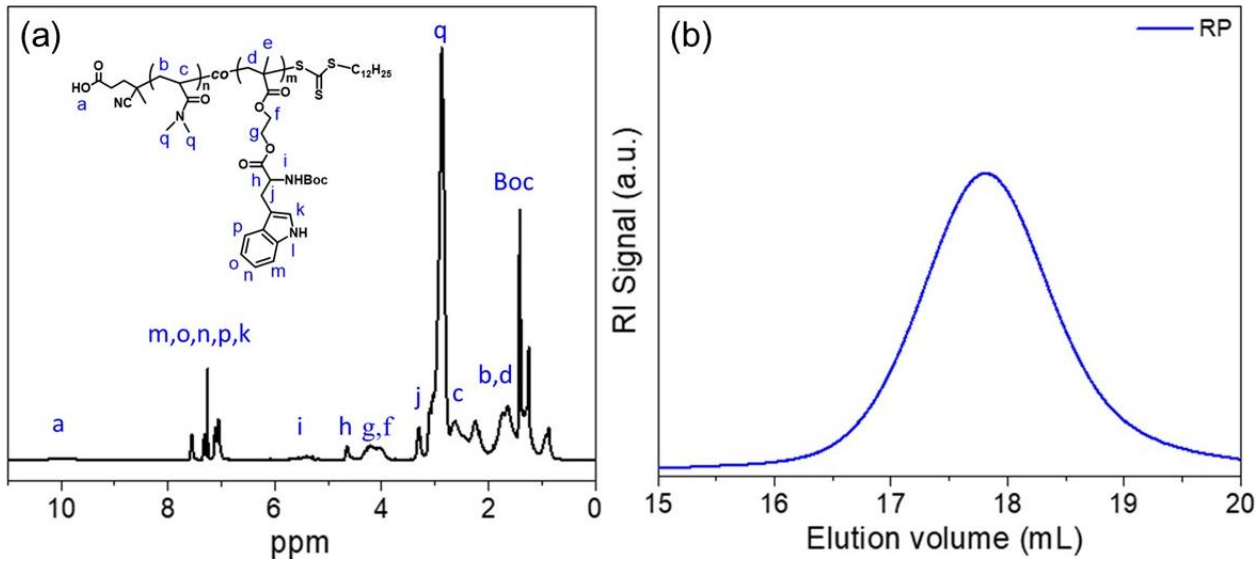

Figure S1. (a) ${ }^{1} \mathrm{H}$ NMR spectrum of $\mathrm{P}(\mathrm{DMA}-\mathrm{co}-\mathrm{BTE})(\mathbf{R P})$ in $\mathrm{CDCl}_{3}$. (b) SEC RI traces of RP.

Table S1. Results from the RAFT-mediated copolymerization of DMA and BTE at $70{ }^{\circ} \mathrm{C}$ in DMF.

\begin{tabular}{|c|c|c|c|c|c|}
\hline Polymera & Conv. $^{\text {b }}(\%)$ & BTE content in copolymer $(\mathrm{mol} \%)^{\mathrm{c}}$ & $M_{\mathrm{n}, \text { theo }}{ }^{\mathrm{d}}\left(\mathrm{g} \mathrm{mol}^{-1}\right)$ & $M_{\mathrm{n}, \mathrm{SEC}}{ }^{\mathrm{e}}\left(\mathrm{g} \mathrm{mol}^{-1}\right)$ & $\boldsymbol{Ð}^{\mathrm{e}}$ \\
\hline $\mathbf{R P}$ & 75 & 12 & 15,000 & 13,700 & 1.19 \\
\hline
\end{tabular}

${ }^{\mathrm{a}}$ Copolymerization was carried out by maintaining the ratio of $\left.[\mathrm{DMA}+\mathrm{BTE}] /[\mathrm{CDP}] /[\mathrm{AIBN})\right]=150: 1: 0.05$ for $6 \mathrm{~h} .{ }^{\mathrm{b}}$ Monomer conversion as obtained by gravimetric analysis on the basis of the amount of monomer charged. ${ }^{c}$ Determined by ${ }^{1} \mathrm{H}$ NMR spectroscopy. ${ }^{\mathrm{d}} M_{\mathrm{n}, \text { theo }}=(([\mathrm{DM} A$ $+\mathrm{BTE}] /[\mathrm{CDP}] \times$ conversion $\times$ average molecular weight $(\mathrm{MW})$ of monomer $)+(\mathrm{MW}$ of $\mathrm{CDP})) .{ }^{\mathrm{e}}$ Determined from SEC analysis.

\section{Synthesis of dansyl-tagged copolymer P(MMA-co-(Ala-HEMA)-co-(Dansyl-Ala-}

\section{HEMA)) (DCP)}

\subsection{Synthesis of $\mathrm{P(MMA-co-Boc-Ala-HEMA),} \mathrm{BCP}$}

The copolymerization reactions of MMA with Boc-Ala-HEMA were carried out at $70{ }^{\circ} \mathrm{C}$ via $\mathrm{RAFT}$ method by using CDP as the RAFT agent with [MMA + Boc-Ala-HEMA]/[CDP]/[AIBN] = 100: 1: 0.2 following a previously developed copolymerization procedure by our group. ${ }^{5}$

\subsection{Deprotection of Boc Groups}

To obtain the free primary amine-containing polymer, $\mathrm{P}\left(\mathrm{MMA}-\mathrm{co}-\left(\mathrm{NH}_{3}{ }^{+}\right.\right.$-Ala-HEMA)), the Bocprotected polymer (BCP) was treated with TFA in DCM at room temperature. ${ }^{5-6}$ In a classic experiment, $\mathbf{B C P}$ $(500 \mathrm{mg})$ was taken in a vial $(20 \mathrm{~mL})$, and solubilized in $1.5 \mathrm{~mL}$ of DCM. To ensure homogeneity, the solution was stirred for $10 \mathrm{~min}$. Then, $1.5 \mathrm{~mL}$ of TFA was dropwise added to the mixture at $5{ }^{\circ} \mathrm{C}$, and the reaction was further stirred for $4 \mathrm{~h}$ at room temperature. The resulting Boc-deprotected copolymer, BCP was isolated by 


\section{Supporting Information}

precipitation from diethyl ether. Finally, the copolymer was dried under vacuum for $12 \mathrm{~h}$ and isolated as a yellow powder.

\subsection{Dansyl Group Labelling, P(MMA-co-(Ala-HEMA)-co-(Dansyl-Ala-HEMA)), DCP}

The dansyl labelling was achieved by the treatment of BCP with dansyl chloride in presence of triethylamine following the previously reported technique. ${ }^{5}$
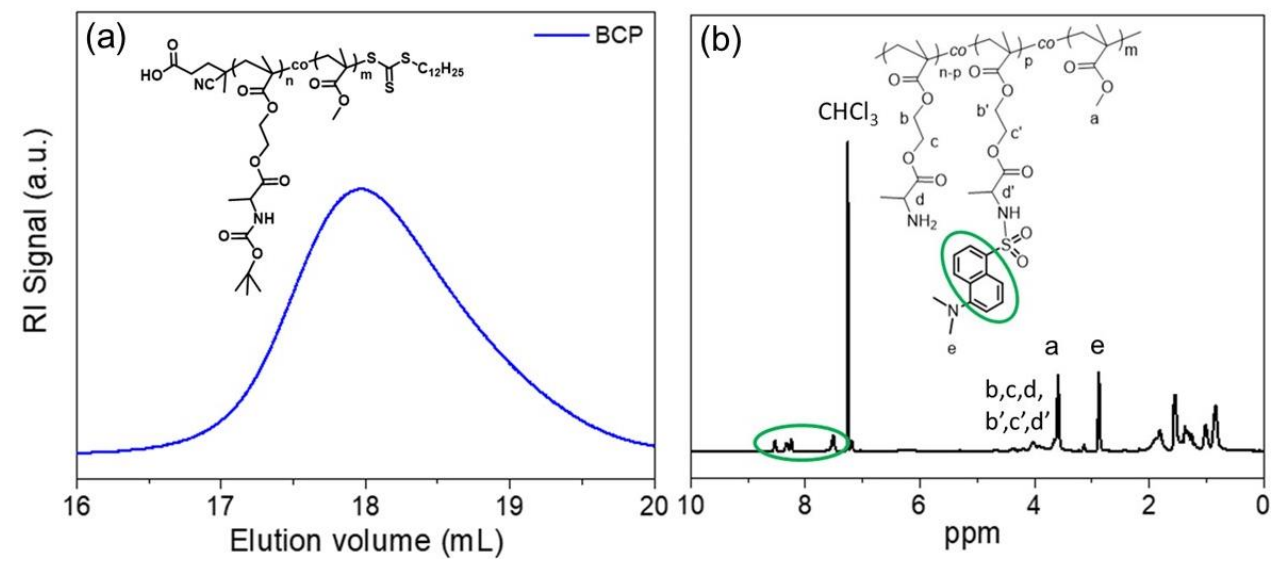

Figure S2. (a) SEC RI traces of BCP (b) The ${ }^{1} \mathrm{H}$ NMR spectra of dansyl tagged copolymer (DCP) in $\mathrm{CDCl}_{3}$.

Table S2. Experimental results from the RAFT copolymerization of MMA and Boc-Ala-HEMA in DMF at $70{ }^{\circ} \mathrm{C}$ for $8 \mathrm{~h}$.

\begin{tabular}{|c|c|c|c|c|c|}
\hline Polymer & Conv. $^{\mathrm{a}}(\%)$ & Boc-Ala-HEMA content in copolymer $(\mathrm{mol} \%)^{b}$ & $M_{\mathrm{n}, \text { theo }}{ }^{\mathrm{c}}(\mathrm{g} / \mathrm{mol})$ & $M_{\mathrm{n}, \mathrm{SEC}}{ }^{\mathrm{d}}(\mathrm{g} / \mathrm{mol})$ & $\boldsymbol{D}^{\mathrm{d}}$ \\
\hline BCP & 60 & 20 & 15,600 & 10,600 & 1.21 \\
\hline
\end{tabular}

${ }^{\mathrm{a}}$ Determined by gravimetric analysis on the basis of the amount of monomer feed. ${ }^{\mathrm{b}}$ Determined from ${ }^{1} \mathrm{H}$ NMR analysis. ${ }^{c} M_{n, \text { theo }}=$ $(([$ Monomer $] /[\mathrm{CDP}] \times$ average molecular weight $(\mathrm{MW})$ of monomer $\times$ conversion $)+(\mathrm{MW}$ of CDP $)) .{ }^{\mathrm{d}}$ Determined from SEC analysis.

\section{Design of FRET Pair and Sensing Study with NACs}

The optimized FRET pair of polymer-polymer was designed by mixing the different concentration of $\mathbf{R P}$ (donor) and DCP (acceptor) from their stock solutions, that is $1 \mathrm{mg} / \mathrm{ml}\left(\approx 10^{-4} \mathrm{M}\right)$ in THF solvent. In order to avoid self-quenching, solutions were further diluted to shape the novel FRET pair. Finally, we established the donor-acceptor ratio of our FRET pair to $1.05 \mu \mathrm{M}$ of $\mathbf{R P}$ and $5.89 \mu \mathrm{M}$ of DCP and applied it further for sensing studies of NACs. Here, sensing analytes are DNT, TNT, and TNP, whose stock solutions were also prepared in THF solvent of known concentration 10.98, 4.4 and $2.2 \mathrm{mM}$, respectively. Next, sensing studies were performed through fluorescence quenching titration experiments with the addition of aliquots of NACs into the optimized FRET pair solution. Both the excitation and emission spectra (excited at the peak maxima of donors' absorbance 


\section{Supporting Information}

spectra) were recorded for $3 \mathrm{~mL}$ solution of FRET pair before and after adding the aliquots of analyte solution, gradually. All these spectra were recorded in a $10 \times 10 \mathrm{~mm}$ quartz cuvette.
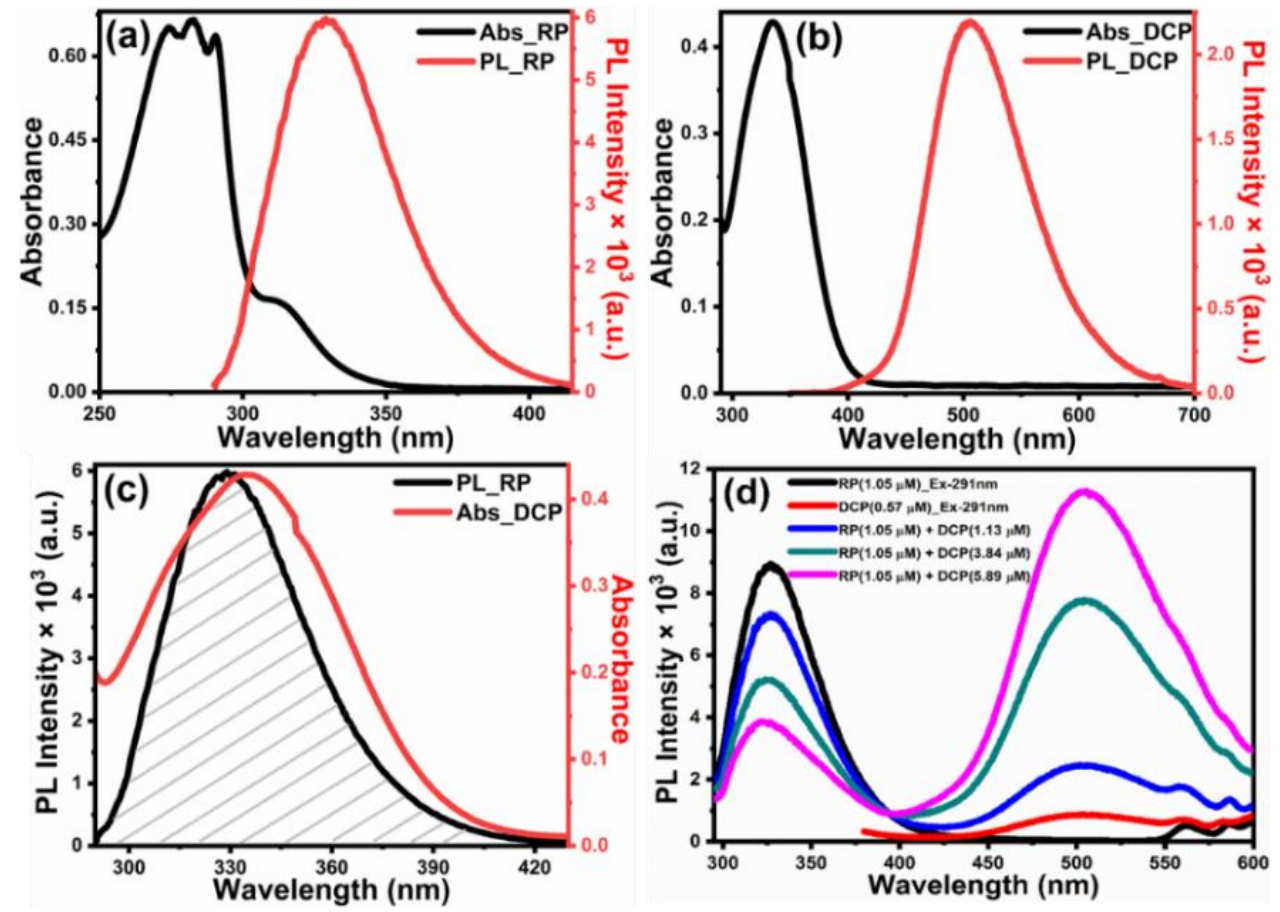

Figure S3. Absorbance and emission spectra of (a) Tryptophan-based copolymer RP (b) Dansyl-based copolymer DCP (c) Spectral overlap of PL of RP and Abs of DCP (d) FRET signal for donor-acceptor pair at the excitation wavelength of donor (291 nm).

\section{Quantum Yield Calculation}

The quantum yield was calculated using the following equation (S3):

$$
\Phi_{u}=\Phi_{s} \frac{F_{u}\left(O D_{s}\right) n_{u}^{2}}{F_{s}\left(O D_{u}\right) n_{s}^{2}}
$$

where, $\boldsymbol{\Phi}$ is the quantum yield (QY), $F$ is the integrated area under the corrected emission spectrum, OD is the absorbance at the excitation wavelength, $\mathrm{n}$ is the refractive index (RI) of the solution and the subscripts $\mathrm{u}$ and $\mathrm{s}$ refer to the unknown and the standard, respectively. Quinine Sulphate has QY of 0.54 in $0.1 \mathrm{M} \mathrm{H}_{2} \mathrm{SO}_{4}(\mathrm{RI}=1.33){ }^{2}$ 


\section{Supporting Information}
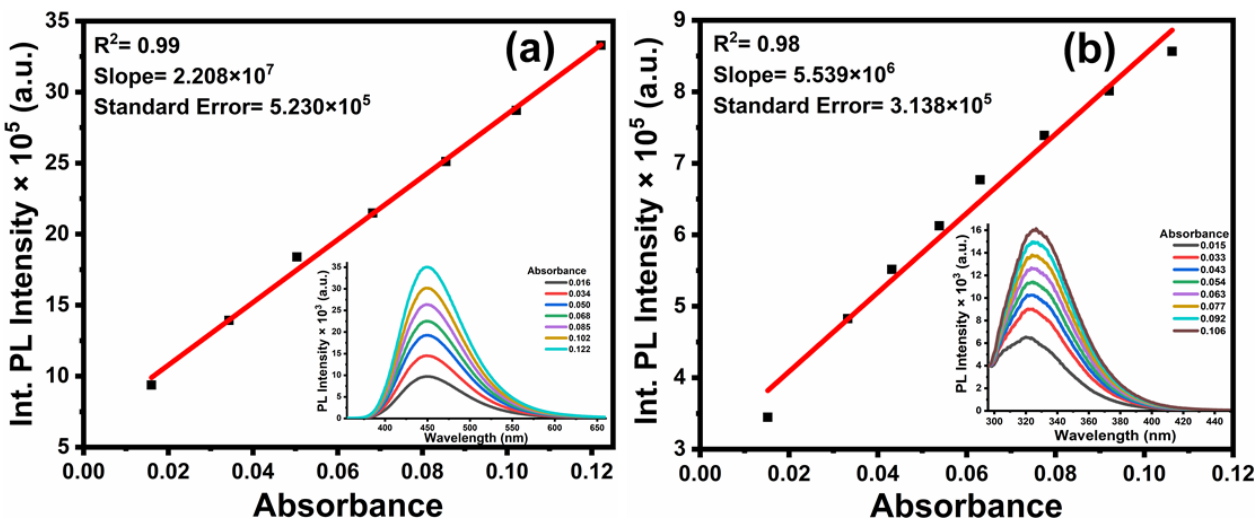

Figure S4. Plots showing linear relationship between integral photoluminescence (PL) intensity at respective absorbances for (a) Quinine Sulphate and (b) tryptophan based copolymer RP (Inset plots showing recorded photoluminescence spectra with different absorbances).

The parameter $\boldsymbol{F}_{\boldsymbol{u}} /\left(\boldsymbol{O} \boldsymbol{D}_{\boldsymbol{u}}\right)$ and $\boldsymbol{F}_{\boldsymbol{s}} /\left(\boldsymbol{O} \boldsymbol{D}_{\boldsymbol{s}}\right)$ corresponds to the slope of plots showing a linear relationship between integral photoluminescence (PL) intensity at respective absorbances for unknown and the standard, respectively. The linear plots of integrated PL intensities against selected absorbances in the range of 0.01 to 0.1 are shown in Figure S4. ${ }^{7}$ So, the relative fluorescence quantum yield (\%) of tryptophan based copolymer RP was obtained to be $15.2 \pm 0.9$, determined by the comparative method using a Quinine Sulphate standard. ${ }^{8}$
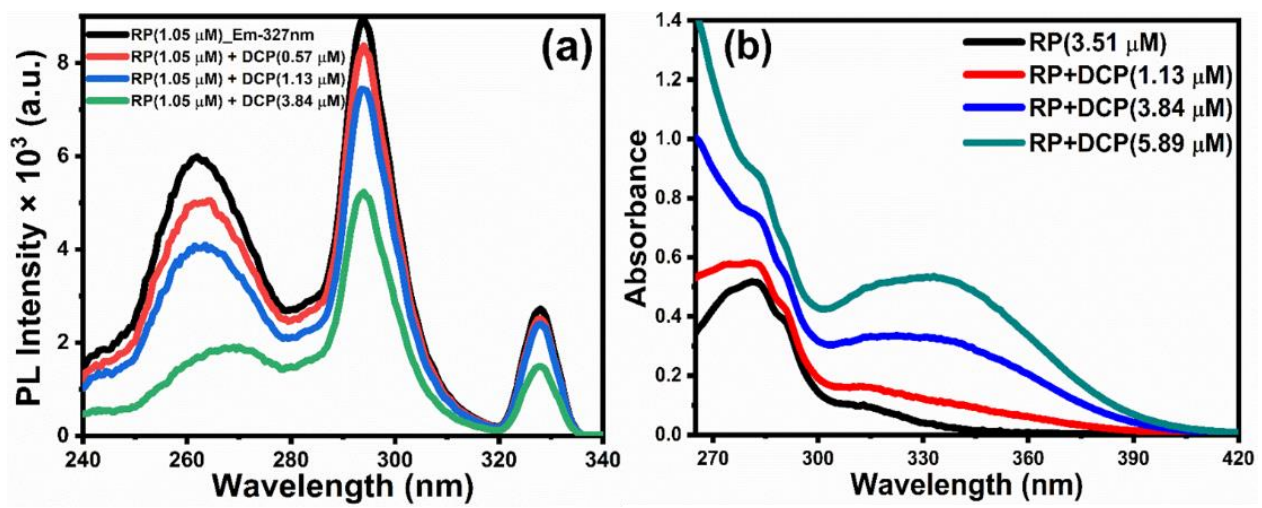

Figure S5. (a) Excitation (at emission wavelength of donor) and (b) UV-Vis absorbance spectra of RP (donor)- DCP (acceptor) complex at different concentration ratio.

\section{Calculation of FRET Pair parameters}

The Förster distance $\left(R_{0}\right)$, distance at which energy transfer efficiency is $50 \%$, is distinctive for a specific donor-acceptor pair and defined by equation (S4).

$$
R_{0}=0.211\left[K^{2} n^{-4} \Phi_{D} J_{\lambda}\right]^{1 / 6}
$$




\section{Supporting Information}

where $K^{2}$ is the dipole orientation factor, having value range from 0 (perpendicular transition dipoles) to 4 (collinear dipoles). Here, $K^{2}=0$ stand for the complete absence of ultrafast component and ultimately forbidden the FRET process. As our FRET system perceived an ultrafast component, therefore, for the calculation of $R_{0}$, we applied an orientational averaging value of $K^{2}=2 / 3$, considering the random orientation of dipoles. $n$ is the refractive index of the sensory medium, e.g., $n=1.407$ for THF. $\Phi_{D}$ is the PLQY of the only donor.

The spectral overlap integral $J(\lambda)$ between the emission spectra of donor and the excitation spectra of acceptor molecule can be calculated as

$$
J(\lambda)=\int_{0}^{\infty} F_{D}(\lambda) \varepsilon_{A}(\lambda) \lambda^{4} d \lambda
$$

where $F_{D}(\lambda)$ and $\varepsilon_{A}(\lambda)$ are the normalized emission spectra of donor and molar excitation coefficient of acceptor at wavelength $\lambda$ (in $\mathrm{nm}$ ).

\section{Calculation of Radiative and Non-Radiative decay rate}

The observed average lifetime ( $\left.\tau_{\text {avg }}\right)$ can be combined with the fluorescence quantum yield $\left(\Phi_{D}\right)$ to determine the radiative $\left(1 / \tau_{\mathrm{r}}\right)$ and non-radiative $\left(1 / \tau_{\mathrm{nr}}\right)$ decay rate of the relevant states for both Donor and Acceptor using the following equations:

$$
\begin{array}{r}
1 / \tau_{r}=\Phi_{D} / \tau_{\text {avg }} \\
1 / \tau_{n r}=1 / \tau_{\text {avg }}-1 / \tau_{r}
\end{array}
$$

Table S3. Calculated values of radiative and non-radiative decay rate for donor and acceptor.

\begin{tabular}{|c|c|c|c|c|c|}
\hline Components & Quantum Yield $\left(\Phi_{\boldsymbol{D}}\right)$ & Avg. Lifetime $\left(\boldsymbol{\tau}_{\text {avg }}\right)(\mathbf{n s})$ & $\left(\mathbf{1} / \boldsymbol{\tau}_{\text {avg }}\right)\left(\mathbf{n s}^{-1}\right)$ & $\left(\mathbf{1} / \boldsymbol{\tau}_{\mathbf{r}}\right)\left(\mathbf{n s}^{-1}\right)$ & $\left(\mathbf{1} / \boldsymbol{\tau}_{\mathbf{n r}}\right)\left(\mathbf{n s}^{-1}\right)$ \\
\hline RP & 0.15 & 1.9 & 0.510 & 0.078 & 0.432 \\
\hline DCP & $0.77^{\$}$ & 13.3 & 0.075 & 0.058 & 0.017 \\
\hline
\end{tabular}

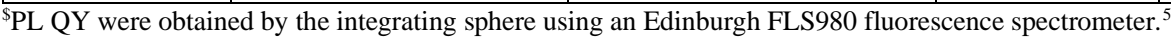

\section{Electrochemical Properties Study (Cyclic Voltammetry Study)}

To ascertain the redox propensity of the copolymer, cyclic voltammetry measurements were performed for the copolymer (RP and $\mathbf{D C P})$ in acetonitrile. The representative cyclic voltammetry was measured with a computer-controlled Princeton Applied Research 263A electrochemical workstation using platinum (Pt) disk as a working electrode, $\mathrm{Pt}$ wire as the counter electrode and $\mathrm{Ag} / \mathrm{AgNO}_{3}$ (in acetonitrile) as the reference electrode, 


\section{Supporting Information}

scan rate $50 \mathrm{mV} / \mathrm{s}$. The $0.1 \mathrm{M}$ tetrabutylammonium hexafluorophosphate (TBAHFP) was used as supporting electrolyte and ferrocene as an internal standard. In case of RP and DCP, cyclic voltammetry run shows an oxidation peak at $+1.32 \mathrm{~V}$ and $+0.89 \mathrm{~V}$, respectively. The reduction peak at $-1.21 \mathrm{~V}$ is due to presence of water in acetonitrile. Generally, the RP and DCP copolymers posse low oxidation potentials attesting the electron-richness of the tryptophan and dansyl unit, respectively.
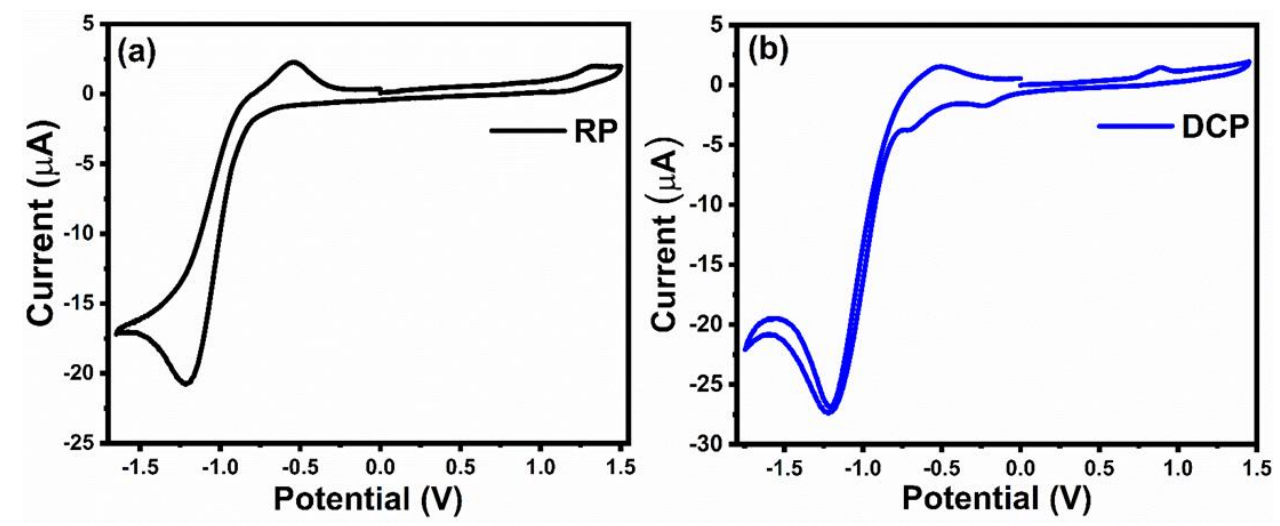

Figure S6. Cyclic voltammograms recorded for (a) RP (donor) (b) DCP (acceptor) in acetonitrile.

Table S4. Electrochemical analysis of copolymers.

\begin{tabular}{|c|c|c|c|}
\hline Copolymers & HOMO $(\mathbf{e V})^{\mathbf{a}}$ & LUMO $(\mathbf{e V})^{\mathbf{b}}$ & ${\text { Band gap }\left(\mathbf{E}_{\mathbf{0}-0)}\right)(\mathbf{e V})^{\mathbf{c}}}^{\text {HP }}$ \\
\hline RPP & -6.12 & -2.04 & 4.08 \\
\hline
\end{tabular}

${ }^{\mathrm{a}} \mathrm{HOMO}=-\mathrm{e}\left[4.8+\mathrm{E}_{\mathrm{ox}}\right] .{ }^{\mathrm{b}} \mathrm{LUMO}=\mathrm{HOMO}+\mathrm{E}_{0-0 .}{ }^{\mathrm{c}}$ Optical band gap obtained from the intersection of normalized absorption and emission spectra.

Table S5. Frontier molecular orbitals (HOMO and LUMO) energy for Nitroaromatics.

\begin{tabular}{|c|c|c|c|}
\hline Analytes & HOMO (eV) & LUMO (eV) & Band gap $(\mathrm{eV})$ \\
\hline 2,4-Dinitrotoluene (DNT) & -8.347 & -3.468 & 4.878 \\
\hline 2,4,6-Trinitrotoluene (TNT) & -8.743 & -4.029 & 4.713 \\
\hline 2,4,6-Trinitrophenol (TNP) & -8.808 & -4.143 & 4.665 \\
\hline
\end{tabular}

"HOMO and LUMO energies are calculated by DFT, frontier orbital theory using B3LYP functional and polarized 6-31G/basis set. ${ }^{5}$

\section{Calculation of Detection Limit}

The limit of detection (LOD) was estimated using the equation $3 \sigma / \mathrm{K}$, where $\sigma$ denotes the standard deviation of intensity response and $\mathrm{K}$ represents the slope of the calibration curve.

Table S6. Limit of detection (LOD) values obtained for various analytes.

\begin{tabular}{|c|c|}
\hline Analytes & Detection Limit \\
\hline 2,4-Dinitrotoluene (DNT) & $5.4 \mu \mathrm{M}(0.98 \mathrm{ppm})$ \\
\hline $\mathbf{2 , 4 , 6}$-Trinitrotoluene (TNT) & $2.3 \mu \mathrm{M}(0.53 \mathrm{ppm})$ \\
\hline $\mathbf{2 , 4 , 6 - T r i n i t r o p h e n o l ~ ( T N P ) ~}$ & $0.4 \mu \mathrm{M}(0.1 \mathrm{ppm})$ \\
\hline
\end{tabular}




\section{Supporting Information}

Table S7. A comparative study of some conjugated polymers-based reports for Nitroaromatic explosive detection.

\begin{tabular}{|c|c|c|c|c|c|}
\hline Publications & $\mathrm{K}_{\text {SV }}(\mathrm{M}-1)$ & Detection Limit & $\begin{array}{c}\text { Linear range of } \\
\text { detection }\end{array}$ & Selectivity & Sensing Mechanism \\
\hline Present Manuscript & $\begin{array}{l}6.3 \times 10^{3}(\mathrm{DNT}) \\
7.6 \times 10^{3}(\mathrm{TNT}) \\
2.2 \times 10^{4}(\mathrm{TNP}) \\
\end{array}$ & $\begin{array}{l}5.4 \mu \mathrm{M}(\mathrm{DNT}) \\
2.3 \mu \mathrm{M}(\mathrm{TNT}) \\
0.4 \mu \mathrm{M}(\mathrm{TNP}) \\
\end{array}$ & $\begin{array}{l}\text { 36.5-285.3 } \mu \mathrm{M} \text { (DNT) } \\
14.6-142.0 \mu \mathrm{M} \text { (TNT) } \\
0.7-57.4 \mu \mathrm{M} \text { (TNP) }\end{array}$ & Selective & $\begin{array}{l}\text { Electron and Energy } \\
\text { Transfer }\end{array}$ \\
\hline $\begin{array}{c}\text { Langmuir, 2020, } 36(6), \\
1462-1466\end{array}$ & NA & $\begin{array}{l}1.4 \mathrm{ppm}(\mathrm{TNT}) \\
8.4 \mathrm{ppm}(\mathrm{DNB}) \\
249.5 \mathrm{ppm}(\mathrm{NB}) \\
\end{array}$ & (1) & Selective & $\begin{array}{l}\text { Non-fluorescent } \\
\text { Electron Transfer } \\
\text { Complex }\end{array}$ \\
\hline $\begin{array}{c}\text { Scientific reports, 2019, } \\
9(1), 7269\end{array}$ & $\begin{array}{l}1.1 \times 10^{3}(\mathrm{DNT}) \\
1.3 \times 10^{3}(\mathrm{TNT}) \\
1.6 \times 10^{4}(\mathrm{TNP})\end{array}$ & $\begin{array}{l}10.1 \mu \mathrm{M}(\mathrm{DNT}) \\
9.1 \mu \mathrm{M}(\mathrm{TNT}) \\
3.7 \mu \mathrm{M}(\mathrm{TNP})\end{array}$ & $\begin{array}{l}\text { 36.5-621.6 } \mu \mathrm{M} \text { (DNT) } \\
29.2-498.4 \mu \mathrm{M} \text { (TNT) } \\
14.5-247.1 \mu \mathrm{M} \text { (TNP) }\end{array}$ & Selective & Electron Transfer \\
\hline $\begin{array}{c}\text { Nanoscale, 2014,6, } \\
2608-2612\end{array}$ & $\begin{array}{c}2.36 \times 10^{3} \\
(\mathrm{DNT})\end{array}$ & $0.34 \mathrm{mM}(\mathrm{DNT})$ & $0.05-5 \mathrm{mM}(\mathrm{DNT})$ & NA & Electron Transfer \\
\hline $\begin{array}{c}\text { Nanoscale, 2015,7, } \\
1872-1878\end{array}$ & NA & $0.30 \mu \mathrm{M}(\mathrm{TNP})$ & 1-60 $\mu \mathrm{M}(\mathrm{TNP})$ & Selective & $\begin{array}{l}\text { Electrostatic } \\
\text { Interaction }\end{array}$ \\
\hline $\begin{array}{c}\text { Analyst, 2018, 143, } \\
\text { 1036-1041 }\end{array}$ & $\begin{array}{l}3.1 \times 10^{3}(\mathrm{DNT}) \\
9.1 \times 10^{3}(\mathrm{TNT})\end{array}$ & $\begin{array}{l}7.6 \mu \mathrm{M}(\mathrm{DNT}) \\
2.5 \mu \mathrm{M}(\mathrm{TNT})\end{array}$ & $0-10$ ppm (TNT) & Selective & $\begin{array}{l}\text { Aggregation-induced } \\
\text { emission } \\
\text { enhancement (AIEE) }\end{array}$ \\
\hline $\begin{array}{c}\text { Sensors and Actuators, } \\
\text { B 2018, 262, 298-305 }\end{array}$ & $\begin{array}{l}1.42 \times 10^{5} \\
(\mathrm{TNP})\end{array}$ & $0.7 \mu \mathrm{M}(\mathrm{TNP})$ & $0-100 \mu \mathrm{M}(\mathrm{TNP})$ & Selective & $\begin{array}{c}\text { Molecular } \\
\text { Interactions } \\
\end{array}$ \\
\hline $\begin{array}{c}\text { Anal. Chem., 2007, 79, } \\
\text { 6, 2386-2393 }\end{array}$ & NA & $\begin{array}{l}96.86 \mu \mathrm{M} \\
(\mathrm{TNT})\end{array}$ & NA & NA & SPME-GC/IRMS \\
\hline
\end{tabular}
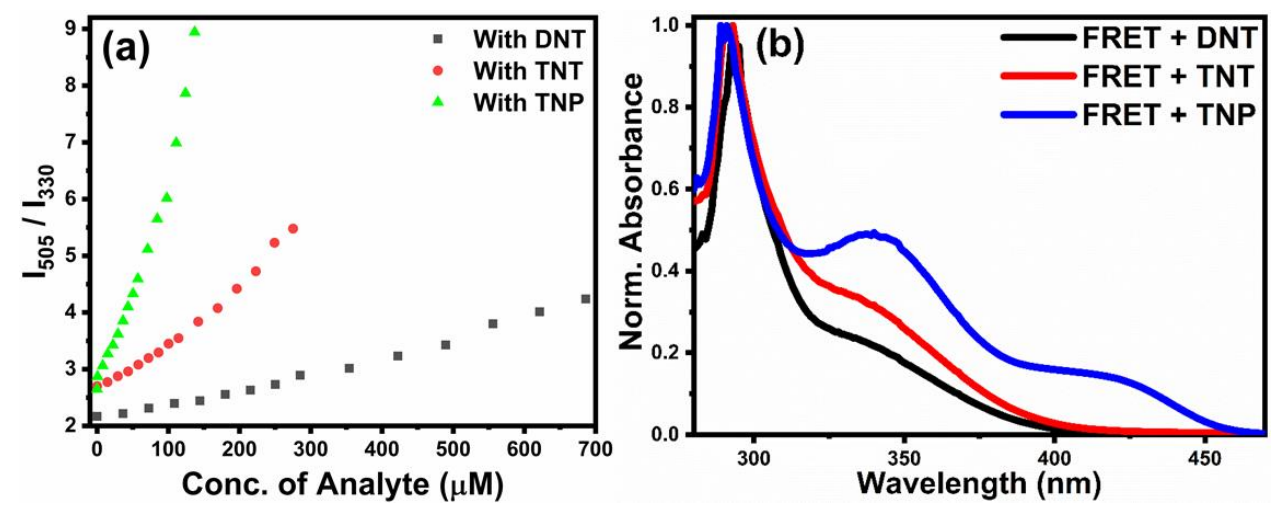

Figure S7. (a) Plot of fluorescence emission intensity ratio of DCP and $\mathbf{R P}\left(I_{505} / I_{330}\right)$ against the increased concentration of analyte $[Q]$ and (b) UV-Vis absorbance spectra of the FRET pair with different NACs
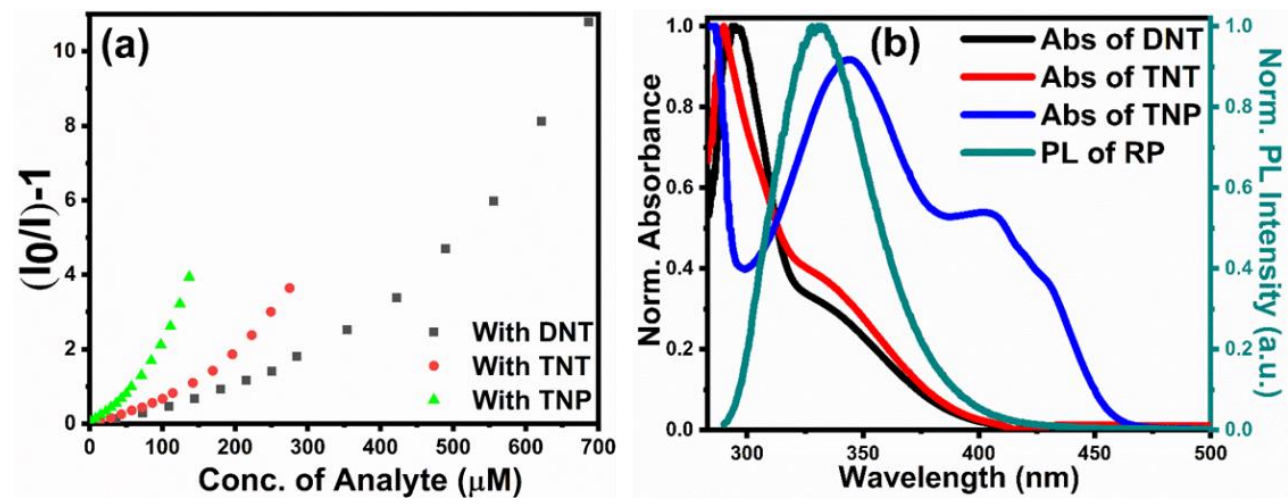

Figure S8. (a) Stern-Volmer plot for fluorescence emission intensity change at $505 \mathrm{~nm}\left(\mathrm{I}_{0} / \mathrm{I}-1\right) \mathrm{v} / \mathrm{s}$ concentration of analyte $[Q]$ and (b) Spectral overlap of the Norm. emission spectra of RP with Norm. absorbance spectra of different NACs. 


\section{Supporting Information}

\section{Ultrafast Transient Absorption (TA) spectroscopic study}

Table S8. Tri-exponential fitting parameters (relative amplitude and lifetime) for the exciton decay kinetics of copolymer DCP.

\begin{tabular}{|c|c|c|c|}
\hline Wavelength (nm) & $\mathbf{5 3 4}$ & $\mathbf{6 7 8}$ & $\mathbf{7 7 4}$ \\
\hline $\mathbf{A 1}$ & 0.75 & 0.58 & 0.32 \\
\hline $\mathbf{t 1}$ (ps) & 0.65 & 0.46 & 2.89 \\
\hline $\mathbf{A 2}$ & 0.10 & 0.17 & 0.42 \\
\hline $\mathbf{t 2}$ (ps) & 87 & 204 & 366 \\
\hline $\mathbf{A 3}$ & -0.15 & 0.25 & 0.26 \\
\hline $\mathbf{t 3}$ (ps) & 7040 & 7070 & 4010 \\
\hline
\end{tabular}

Table S9. Tri-exponential fitting parameters (relative amplitude and lifetime) for the exciton decay kinetics of copolymer DCP in presence of DNT

\begin{tabular}{|c|c|c|c|}
\hline Wavelength (nm) & $\mathbf{5 0 8}$ & $\mathbf{6 6 0}$ & $\mathbf{7 6 4}$ \\
\hline $\mathbf{A 1}$ & 0.96 & 0.62 & 0.52 \\
\hline $\mathbf{t 1}$ (ps) & 0.19 & 0.40 & 0.99 \\
\hline $\mathbf{A 2}$ & 0.02 & 0.28 & 0.37 \\
\hline $\mathbf{t 2}$ (ps) & 9.56 & 17.30 & 17.10 \\
\hline $\mathbf{A 3}$ & 0.02 & 0.10 & 0.11 \\
\hline $\mathbf{t 3}$ (ps) & 6000 & 8750 & 6490 \\
\hline
\end{tabular}

Table S10. Tri-exponential fitting parameters (relative amplitude and lifetime) for the exciton decay kinetics of copolymer DCP in presence of TNT.

\begin{tabular}{|c|c|c|c|}
\hline Wavelength (nm) & $\mathbf{5 4 5}$ & $\mathbf{6 2 6}$ & $\mathbf{7 6 7}$ \\
\hline $\mathbf{A 1}$ & 0.25 & 0.18 & 0.45 \\
\hline $\mathbf{t 1}(\mathbf{p s})$ & 11.40 & 70 & 400 \\
\hline $\mathbf{A 2}$ & 0.70 & 0.56 & 0.28 \\
\hline $\mathbf{t 2}$ (ps) & 0.31 & 0.81 & 5.38 \\
\hline $\mathbf{A 3}$ & -0.05 & 0.26 & 0.27 \\
\hline $\mathbf{t 3}$ (ps) & 3570 & 12100 & 4380 \\
\hline
\end{tabular}

Table S11. Tri-exponential fitting parameters (relative amplitude and lifetime) for the exciton decay kinetics of copolymer DCP in presence of TNP.

\begin{tabular}{|c|c|c|c|}
\hline Wavelength (nm) & $\mathbf{6 0 9}$ & $\mathbf{6 6 8}$ & $\mathbf{7 2 2}$ \\
\hline $\mathbf{A 1}$ & 0.36 & 0.30 & 0.73 \\
\hline $\mathbf{t 1}$ (ps) & 237 & 106 & 704 \\
\hline $\mathbf{A 2}$ & -0.17 & -0.20 & 0.08 \\
\hline $\mathbf{t 2}$ (ps) & 11.8 & 9.57 & 1.42 \\
\hline $\mathbf{A 3}$ & 0.47 & 0.50 & 0.19 \\
\hline $\mathbf{t 3}$ (ps) & 4580 & 1080 & 18400 \\
\hline
\end{tabular}




\section{Supporting Information}

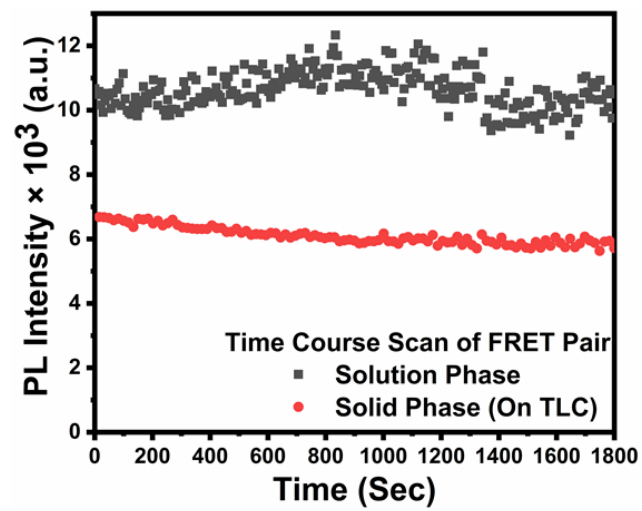

Figure S9. Photodegradation study of FRET probe $\left(\lambda_{\mathrm{Ex}}=291 \mathrm{~nm}, \lambda_{\mathrm{Em}}=505 \mathrm{~nm}\right)$.

\section{Visual Detection of NACs and It's Reversibility}

The reversibility of the sensor probe to NACs was conducted with ethanol (Assay: 99.0\%). As shown in Figure S9, the quenching loss caused by the adsorption of $10 \mu \mathrm{L}$ DNT from the stock solution (under $254 \mathrm{~nm}$ UV illumination) will get recovered moderately after adsorption of $10 \mu \mathrm{L}$ ethanol, even after the five continuous iterations.

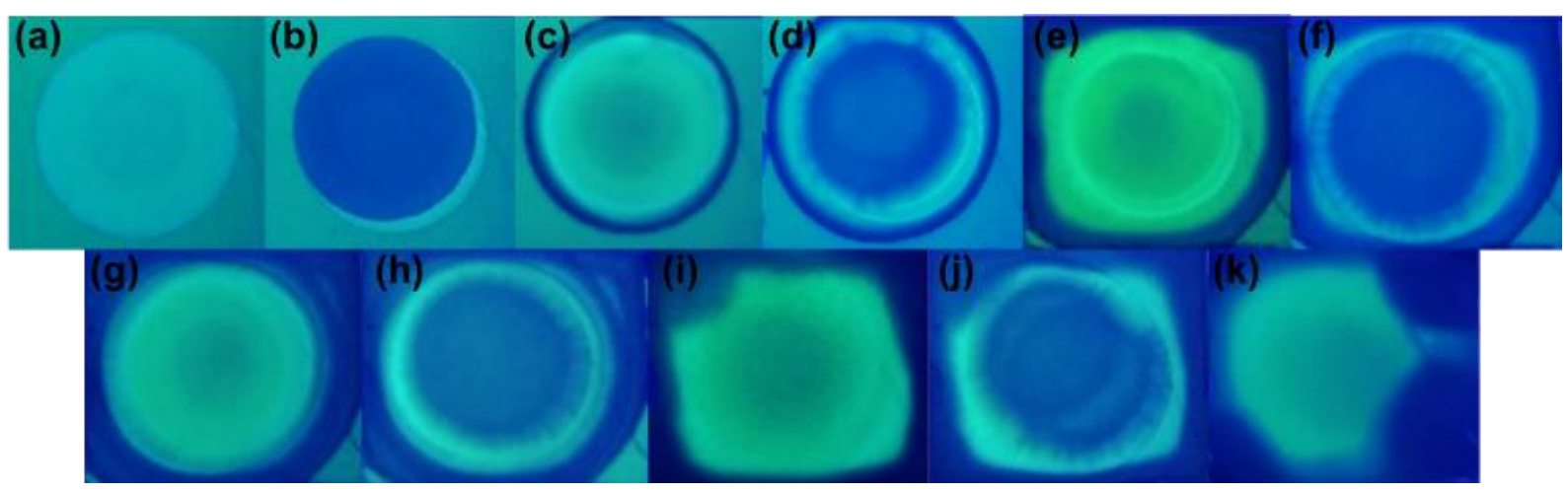

Figure S10. Optical images of test strips (a) unserved sensor (b) to (k) treated with 10 $\mu \mathrm{L}$ DNT (10.98 mM) followed with $10 \mu \mathrm{L}$ ethanol for five continuous iterations.

\section{References}

1. Moad, G.; Chong, Y. K.; Postma, A.; Rizzardo, E.; Thang, S. H., Advances in RAFT polymerization: the synthesis of polymers with defined end-groups. Polymer 2005, 46 (19), 8458-8468.

2. Choudhury, N.; Saha, B.; Ruidas, B.; De, P., Dual-Action Polymeric Probe: Turn-On Sensing and Removal of $\mathrm{Hg} 2+$; Chemosensor for HSO4-. ACS Appl. Polym. Mater. 2019, 1 (3), 461-471.

3. Valeur, B., Molecular Fluorescence Princeples And Applications. Wiley-VCH 2001, 2, 3-527.

4. $\quad$ Bauri, K.; Roy, S. G.; De, P., Side-Chain Amino-Acid-Derived Cationic Chiral Polymers by Controlled Radical Polymerization. Macromol. Chem. Phys. 2016, 217 (3), 365-379.

5. Kumar, V.; Maiti, B.; Chini, M. K.; De, P.; Satapathi, S., Multimodal Fluorescent Polymer Sensor for Highly Sensitive Detection of Nitroaromatics. Sci Rep 2019, 9 (1), 7269.

6. Kumar, S.; De, P., Fluorescent labelled dual-stimuli (pH/thermo) responsive self-assembled side-chain amino acid based polymers. Polymer 2014, 55 (3), 824-832. 


\section{Supporting Information}

7. Dutta, R. K.; Kumar, A., Highly Sensitive and Selective Method for Detecting Ultratrace Levels of Aqueous Uranyl Ions by Strongly Photoluminescent-Responsive Amine-Modified Cadmium Sulfide Quantum Dots. Anal. Chem. 2016, 88 (18), 9071-8.

8. Chen, R. F., Fluorescence Quantum Yields of Tryptophan and Tyrosine. Anal. Lett. 1967, l (1), 35-42. 